\title{
PSYCHOLOGICA
}

\section{Comparação da atenção em jogadores de futebol e em não atletas}

Autor(es): $\quad$ Barbosa, Claudia; Montiel, José Maria; Machado, Afonso Antônio; Bartholomeu, Daniel

Publicado por: Imprensa da Universidade de Coimbra

URL

persistente: $\quad$ URI:http://hdl.handle.net/10316.2/43294

DOI: DOI:https://doi.org/10.14195/1647-8606_60-2_8

Accessed : $\quad$ 26-Apr-2023 13:00:50

A navegação consulta e descarregamento dos títulos inseridos nas Bibliotecas Digitais UC Digitalis, UC Pombalina e UC Impactum, pressupõem a aceitação plena e sem reservas dos Termos e Condições de Uso destas Bibliotecas Digitais, disponíveis em https://digitalis.uc.pt/pt-pt/termos.

Conforme exposto nos referidos Termos e Condições de Uso, o descarregamento de títulos de acesso restrito requer uma licença válida de autorização devendo o utilizador aceder ao(s) documento(s) a partir de um endereço de IP da instituição detentora da supramencionada licença.

Ao utilizador é apenas permitido o descarregamento para uso pessoal, pelo que o emprego do(s) título(s) descarregado(s) para outro fim, designadamente comercial, carece de autorização do respetivo autor ou editor da obra.

Na medida em que todas as obras da UC Digitalis se encontram protegidas pelo Código do Direito de Autor e Direitos Conexos e demais legislação aplicável, toda a cópia, parcial ou total, deste documento, nos casos em que é legalmente admitida, deverá conter ou fazer-se acompanhar por este aviso. 


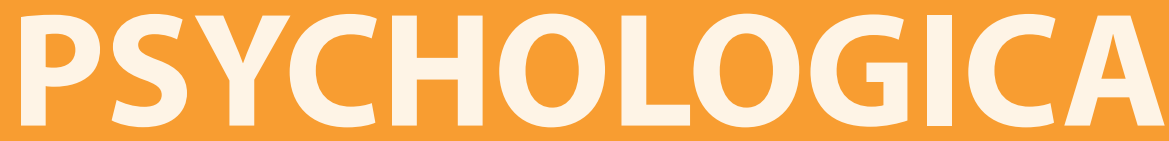




\title{
Comparação da atenção em jogadores de futebol e em não atletas
}

\author{
Claudia Barbosaํㅗ José Maria Montiel², \\ Afonso Antônio Machado ${ }^{3}$ e Daniel Bartholomeu ${ }^{4}$
}

Attention comparison of soccer players and non-athletes

\begin{abstract}
Attentional and motor aspects show great relevance in sports activities. This study aimed to compare the attention of athletes and non-athletes, and check the effects of age on these differences. Participants were 554 males aged between 10-17 years. The instruments used were the Attention Test (a cancellation test) and the evaluation of specific motor skills for football. The results showed that a good performance of the athlete and a good sporting performance are related to a high level of attention to the game and to high motor skills. It is emphasized that individuals with good performance in cancellation tests tend to show better performance in motor skills.
\end{abstract}

Keywords: attention; sporting performance; motor skills

1 Professora e Coordenadora do curso de Psicologia da Faculdade Assis Gurgacz - Cascavel/Paraná. Email: claudia@fag.edu.br

2 Professor do Centro Universitário FIEO - UNIFIEO/SP - Programa de Pós-Graduação Strictu Sensu em Psicologia Educacional - Fundação Instituto de Ensino para Osasco. Email: montieljm@hotmail.com

3 Coordenador do Laboratório de Estudos e Pesquisas em Psicologia do Esporte e do Programa de Pós-graduação em Desenvolvimento Humano e Tecnologias (DEHUTE), no IB/UNESP - Rio Claro. Email: afonsoa@gmail.com

4 Professor do Centro Universitário FIEO - UNIFIEO/SP - Programa de Pós-Graduação Strictu Sensu em Psicologia Educacional - Fundação Instituto de Ensino para Osasco. Email: d_bartholomeu@yahoo.com.br 


\section{Resumo}

Os aspetos atencionais e motores demonstram grande relevância em atividades desportivas. Este estudo teve como objetivo comparar a atenção de atletas e não atletas, e verificar os efeitos da idade nestas diferenças. Participaram 554 indivíduos do sexo masculino na faixa etária entre os 10 e os 17 anos. Os instrumentos utilizados foram o Teste de Atenção por Cancelamento e a avaliação da habilidade motora específica para o futebol. Os resultados indicaram que um bom desempenho do atleta e um bom desempenho desportivo estão relacionados com um elevado nível de atenção ao jogo e de habilidade motora. Ainda pode sublinhar-se que bons desempenhos em testes de atenção por cancelamento tendem a estar relacionados com melhores desempenhos em avaliações da habilidade motora.

Palavras-chave: atenção; desempenho desportivo; habilidade motora

\section{INTRODUÇÃO}

Sternberg (2000) define a atenção como o fenómeno pelo qual se processa ativamente uma determinada quantidade de informação do enorme manancial de informações disponíveis através dos sentidos. A atenção ajuda o indivíduo a monitorizar as interações com o ambiente, mantendo a consciência de adaptação à situação com a qual se depara, relacionando as memórias com a sensação e, assim, possibilitando a continuidade de experiências e ajudando a controlar e planear ações futuras. É por meio do fenómeno da atenção que o indivíduo pode selecionar informações e proceder a um exame mais detalhado das mesmas.

Autores como Bertolucci (2000), Dalgalarrondo (2000), Gazzaniga e Heatherton (2005), Montiel (2005), Sarter, Givens e Bruno (2001) e Sternberg (2000) descrevem uma possível divisão ou classificação dos mecanismos atencionais que é relativa ao tipo de processamento envolvido neste processo, delimitando, assim, os processos atencionais em atenção seletiva, atenção sustentada, atenção alternada e atenção dividida. Cervelló (1999) refere-se a quatro tipos de focos atencionais: amplo interno, amplo externo, estreito interno e estreito externo. Segundo este autor, quando um indivíduo mantém o primeiro tipo de foco, ele é capaz de organizar e integrar um grande número de pensamentos e perceções - é o estilo adequado para analisar e planear ações. O segundo estilo permite ao sujeito explorar, perceber e organizar um grande número de estímulos externos - é o foco adequado perante situações complexas e com um nível grande de informação. O terceiro tipo, estreito interno, auxilia a pessoa a focalizar a atenção numa determinada linha de pensamento - é 
adequado para solucionar problemas concretos ou para meditar. O último estilo atencional, estreito externo, ajuda o indivíduo a focalizar a atenção numa atividade mais ou menos complexa, evitando as distrações com o objetivo de realizar uma determinada ação - é adequado para um grande número de desportos.

Quando esta classificação é aplicada ao contexto desportivo, a amplitude do foco faz referência à quantidade de estímulos aos quais o atleta deve prestar atenção a cada instante (Cervelló, 1999; Andreoni, Kuhn, \& Sprenger, 2015), sendo que o foco amplo está relacionado com um grande número de estímulos e o foco estreito com apenas um ou dois estímulos mais importantes. Já a direção do foco faz referência à orientação da atenção para aspetos externos ou internos ao indivíduo. Exemplificando, no desporto o foco amplo interno pode ser usado para planear uma estratégia, o foco amplo externo é necessário para avaliar uma situação (por exemplo, o posicionamento dos jogadores de defesa), o foco estreito interno é utilizado quando se controla o estado emocional ou se ensaia mentalmente uma tarefa e o foco estreito externo é usado para focalizar poucos sinais externos (exemplo, prestar atenção à bola).

Conforme a descrição de Gould e Damarjian (2000), o processo de prestar atenção a algo envolve três habilidades básicas, que podem estar relacionadas com as classificações anteriormente descritas. A primeira habilidade é a seleção do estímulo relevante no qual se deve focalizar a atenção. A segunda corresponde à manutenção da atenção conforme o ambiente, ou seja, mudança de foco de atenção de acordo com a necessidade. Por último, há a considerar a habilidade de manter a atenção e concentrar-se, ou seja, ficar atento a um estímulo por um determinado período. Por exemplo, um tenista no saque deve decidir o que pretende fazer no ponto (foco interno/amplo), imaginar o saque que pretende fazer (foco interno/estreito) e focalizar o ponto na quadra onde deseja acertar (foco externo/estreito), (Abes, 2004). Segundo Guallar e Pons (1994), pode estar-se alerta de forma transitória (atento a algum estímulo específico, por exemplo, o corredor de $100 \mathrm{~m}$ no atletismo que fica atento ao tiro de largada) ou de forma rotineira (ficar atento durante um longo período de tempo a vários estímulos, por exemplo, no ténis). Martens (1987) aponta que o atleta pode estar atento a estímulos externos ou internos (por exemplo, para controlar os pensamentos negativos).

Em relação aos estudos que envolvam os processos da atenção e/ou atencionais, Solanellas, Front e Rodríguez (1996) conduziram uma pesquisa sobre estilos atencionais com jogadores de ténis, sendo 215 do sexo masculino e 215 do sexo feminino, com idades variando dos 12 anos à idade adulta (34 anos). Os autores utilizaram uma versão adaptada do Test of Attentional and Interpersonal Style (TAIS) de Nideffer (1976) e observaram que a grande maioria dos jogadores usava um foco estreito durante os jogos (83.5\%). Já em relação à direção do foco, os resultados mostraram uma predominância do foco externo (64.6\%), porém com uma diferença significativa entre sexo masculino (58.8\%) e feminino (71.1\%). Mais 
recentemente, Cozza (2008), com objetivo de buscar evidências de validade de instrumentos para a avaliação neuropsicológica das funções executivas em atletas de voleibol, analisou a correlação entre os desempenhos de atletas profissionais em situações de competição, avaliados por meio do scout técnico de vólei e em tarefas que avaliam componentes das funções executivas, e também a correlação entre os desempenhos nas diversas tarefas executivas. Os resultados indicaram correlações positivas significativas entre o scout e o desempenho dos atletas nos Testes de Stroop (Capovilla, Montiel, Macedo, \& Charin, 2005) e Geração Semântica (Capovilla, Capovilla, \& Macedo, 2005) para o total de jogadores. Foram também encontradas correlações entre o desempenho no Teste de Memória de Trabalho Visual (Primi, 2002) e o scout para as posições de levantador, líbero, meio e oposto em pelo menos um dos jogos; entre o desempenho no Teste de Atenção por Cancelamento (Montiel \& Capovilla, 2007) e o scout em um dos sets para a posição de ponta; e entre o teste Torre de Londres (Cozza, 2005) e scout em um dos sets para a posição de líbero. Análises de variância multivariada indicaram diferenças marginais entre as posições em jogo apenas para o tempo de reação de interferência do Teste de Geração Semântica (Cozza, 2008).

Em continuidade no âmbito desportivo, quando se estuda a atenção torna-se necessário encontrar meios de avaliação para poder saber em que situação física e psicológica se encontra um determinado indivíduo e de que maneira a atenção pode afetar, seja positiva ou negativamente, o desempenho do atleta. Como descrito por Guallar e Pons (1994) e Weinberg e Gould (2001), existem vários meios para avaliar a atenção que podem ser aplicados ao contexto do desporto, entre os quais podem citar-se testes, questionários, entrevistas, observações comportamentais e medidas psicofisiológicas, tais como frequência cardíaca e eletroencefalograma (EEG). De entre as variáveis psicofisiológicas, pode salientar-se a importância da frequência cardíaca e da frequência das ondas cerebrais. Com este último procedimento, Radlo, Steinberg, Singer, Barna e Melnikov (2002) avaliaram dois grupos: um que devia realizar uma tarefa de arremesso de dardos mantendo o foco de atenção no alvo (foco externo) e o outro focalizando-se no movimento de arremesso (foco interno). Os resultados indicaram uma desaceleração dos batimentos do coração, mais acentuada instantes antes de executar um arremesso de dardo considerado “ótimo”. Além disso, os autores observaram uma associação entre baixos níveis de atividade alfa no cérebro e bom desempenho.

Para Samulski (2002) as ondas cerebrais detetadas por meio do EEG permitem avaliar se o indivíduo está em estado de vigília, atento ou concentrado. Da mesma forma, Guallar e Pons (1994) relatam, com base em pesquisas na área de psicofisiologia, que, quando o indivíduo focaliza a sua atenção, há uma desaceleração da frequência cardíaca e um aumento da atividade das ondas alfa no hemisfério 
esquerdo do cérebro. Outro aspeto considerado por estes autores foi a utilização de equipamentos para monitorizar os movimentos oculares, pois os olhos realizam permanentemente pequenos movimentos, quando se focaliza a atenção havendo uma fixação do olhar de no mínimo $100 \mathrm{~ms}$. Tais dados foram corroborados por outros estudos (Covre, Macedo, Capovilla, \& Schwartzman, 2005; Covre, Piza, Lukasova, \& Macedo, 2002; Harle \& Vickers, 2001; Vickers, 1996; Vickers, Rodrigues, \& Brown, 2002).

De entre outros estudos relacionando atenção, respostas psicofisiológicas e atividades desportivas, pode destacar-se o de Robazza, Bortoli e Nougier (1998) com atletas de alto nível, do sexo feminino, da seleção italiana, da modalidade de arco e flecha. Nesta pesquisa os autores analisaram cinco tipos de condições: desempenho ótimo, adiamento do disparo, com os olhos fechados, com alta e baixa ativação. Os resultados apresentados demonstraram que a desaceleração da frequência cardíaca era maior na execução 'ótima', de olhos fechados e em condições de simulação, e menor no adiamento do disparo e em situação de alta ativação. Outro estudo, realizado por Shea e Wulf (1999), avaliou a influência sobre a aprendizagem da atenção num foco externo ou interno (grupos 1 e 2) e do feedback de foco externo ou interno (grupos 3 e 4). A tarefa realizada pelos sujeitos era a de se manterem equilibrados sobre um estabilómetro. O grupo 1 prestava atenção nos seus pés (foco interno) e o grupo 2 numa marca fixada no aparelho (foco externo). O grupo 3 recebia um feedback sobre o desvio dos seus pés (feedback de foco interno) e o grupo 4 recebia informações sobre o desvio em relação à marca (feedback de foco externo). Os resultados indicaram uma melhor aprendizagem para os grupos de foco externo.

O estudo de Radlo et al. (2002) foi conduzido com indivíduos que deviam lançar dardos tentando acertar um alvo na parede, sendo os participantes monitorizados quanto à frequência cardíaca e EEG divididos em dois grupos e instruídos a manter um foco interno ou foco externo. Os seus resultados mostraram que os participantes que cometeram menos erros foram aqueles que receberam a orientação entre cada arremesso para manter um foco externo; além disso, eles também apresentaram maior desaceleração dos batimentos do coração e frequência alfa mais baixa no hemisfério esquerdo do cérebro. Outros autores, como Wulf, McConnel, Gärtner e Schwarz (2002), conduziram um estudo com dois experimentos realizados no campo: um com vóleibol e outro com futebol. No primeiro, havia dois grupos de sujeitos de níveis técnicos diferentes, iniciados e avançados e estes eram subdivididos em dois grupos: um grupo recebia um feedback de foco interno e o outro grupo o feedback de foco externo. Neste experimento os jogadores de vólei deviam realizar o saque tipo ténis, na direção de uma área marcada no solo, e recebiam feedback de acordo com o grupo a que pertenciam. Os resultados indicaram que o feedback de foco externo 
influenciava mais positivamente do que o feedback de foco interno na aquisição e melhoria da tarefa motora, tanto em precisão como na mecânica do gesto.

Neste mesmo período, outro estudo, de Wulf, McConnel, Gärtner e Schwarz (2002), foi realizado com dois grupos, foco interno e foco externo, subdivididos de acordo com a frequência com que recebiam o feedback (33\% e 100\%). A atividade era executar passes de futebol de campo para um alvo. Os resultados mostraram que os sujeitos pertencentes aos grupos com feedback de foco externo obtiveram melhores resultados, sendo que a frequência de feedback não apresentou diferenças significativas. No entanto, para os grupos foco interno, os sujeitos que recebiam menos feedback (33\%) desempenharam melhor a atividade. Liao e Masters (2002) estudaram jogadores de hóquei universitários e de básquete iniciados. Os seus resultados demonstraram que o estresse poderia levar os atletas a voltarem-se para si mesmos. Além disso, as duas equipas perderam os confrontos. O outro experimento foi realizado com jogadores iniciados de básquete, em duas fases, divididos em grupo de controlo e experimental, sendo que o grupo experimental foi submetido a situações de estresse, tendo que executar a tarefa em um tempo determinado. A tarefa era a de realizar lances livres. Os resultados indicaram que em situação de estresse os sujeitos com um foco interno de atenção mostraram uma queda no seu desempenho. Tais resultados foram corroborados por Medina-Papst, Bordini e Marques (2016) e, neste estudo, as instruções para o direcionamento do foco de atenção poderiam ser usadas de acordo com o estádio de aprendizagem do sujeito e o objetivo a ser atingido. Assim, as dicas de aprendizagem podem ser estratégias que auxiliam, de maneira simples, os instrutores no treino dos aprendizes, focando aspetos fundamentais da prática.

Sem ter a intenção de esgotar a temática, há a considerar diversas pesquisas que estudam a atenção e os seus componentes envolvidos no contexto do desporto, sugerindo que a atenção está relacionada de maneira significativa com o desempenho de atletas. Por exemplo, Moraes (1990) aponta que um alto nível de ansiedade tende a reduzir a atenção e a concentração durante a execução de uma tarefa ou durante o jogo. Adriano (2003) procurou identificar eventuais variações nos estados atencionais de atletas de futsal em testes aplicados pré e pós-treino. Os resultados revelaram que a pontuação média no teste de atenção aumentou $36.56 \%$ na comparação entre o pré e pós-teste, sugerindo um aumento da atenção com o treino. Becker Jr. (2000) preconiza que antes e durante as competições, quando o nível de ansiedade dos atletas é aumentado, a atenção é reduzida e que, geralmente, a deficiência do jogador na atenção leva à redução no rendimento técnico e tático.

Mais recentemente, Fernandes e Fernandes (2015) investigaram as propriedades psicométricas da adaptação transcultural da versão brasileira do Test of Performance Strategies de Thomas, Murphy e Hardy (1999). Entre os resultados encontrados pelos autores, o controlo atencional, emocional, definição de objetivos, imagética e diálogo 
interno revelaram-se como fatores bem ajustados deste instrumento. Noutra perspetiva, Fechine, Costa, Vasconcelos, Botelho e Carvalho (2015) compararam os níveis de atenção e velocidade percetiva entre idosos praticantes e não praticantes de atividades desportivas. A atenção apresentou diferenças significativas entre idosos praticantes e não praticantes de atividade física, sendo que os praticantes apresentaram níveis superiores de atenção. A velocidade percetiva não diferiu significativamente entre os grupos.

A impulsividade implica uma resposta rápida, sem reflexão e submetida a maior número de erros em decorrência do baixo foco atencional e percetivo na tarefa, afetando o comportamento tático em jogadores de futebol (Cardoso, Machado, \& Teoldo, 2015; García, Rodríguez, \& Garzón, 2011). Esta perspetiva é corroborada por Bonilha, Romero, Sánchez-Ureña e Salas-Cabrera (2015) no seu estudo sobre as características psicológicas de jogadores de futebol e básquete na Costa Rica. Os autores afirmam que os desajustes no foco atencional no desporto implicam rendimentos desportivos deficientes.

Como apresentado anteriormente, a importância da atenção para o desporto pode ser devida a diferentes motivos e apresentar diferentes manifestações. Ladewig (2000) descreve que a aprendizagem de qualquer habilidade motora requer a seleção de informações que podem estar contidas no meio ambiente e/ou serem fornecidas pelos envolvidos nesta relação, tais como o professor ou técnico, e para que esta informação seja retida, para posterior interpretação e possível armazenamento na memória, os processos de atenção são fundamentais. Neste sentido, ao se considerar a atenção como uma habilidade que tem sido relacionada com frequência com o desempenho motor (Andrade, Luft, \& Rolim, 2004; Piek et al., 2004), é importante dispor de instrumentos que apresentem condições satisfatórias para tal avaliação. Neste sentido, torna-se de suma importância buscar evidências de validade de testes para o psicodiagnóstico desportivo e até mesmo desenvolver estudos cujos propósitos tenham o objetivo de verificar a existência de validade, por exemplo, em relação a outras variáveis de testes para avaliar atenção no contexto do desporto, sobretudo no Brasil. Na prática de uma modalidade desportiva, o atleta depende da atenção e da habilidade motora. Os estudos apresentados anteriormente apontam a relevância destas capacidades para o desportista. Assim, este trabalho teve como objetivo comparar a atenção de atletas e não atletas.

Com base nos estudos anteriores, aventa-se que os atletas apresentarão maiores capacidades atencionais do que os não atletas. Uma vez que as habilidades cognitivas tendem a ter uma redução em função da idade, espera-se que também existam diferenças de idade nas medidas de atenção, havendo um efeito de interação entre atletas e não atletas. Finalmente, a terceira hipótese aventada refere-se à associação significativa entre as medidas de desempenho desportivo e de atenção. Estes resultados fornecerão evidências de validade para o teste de atenção em questão (Teste 
de Atenção por Cancelamento de Montiel \& Seabra, 2007), quanto ao desempenho desportivo, uma vez que as relações deste teste com outros instrumentos que avaliam a atenção são apontadas na literatura.

\section{MÉTODO}

\section{Participantes}

Foram avaliados 554 indivíduos do sexo masculino, na faixa etária entre 10 a 17 anos $(M=14.15 \pm D P=2.16)$. Destas, 107 crianças estão na faixa etária dos 10 aos 11 anos (19.3\% da amostra), 156 encontram-se entre os 12 e os 13 anos (28.2\%), 143 com idade entre 14 e 15 anos (25.8\%) e 145 com idade entre 16 e 17 anos (26.2\%). Três sujeitos ( $0.5 \%$ da amostra) não forneceram dados sobre a idade.

Os participantes foram divididos em 2 grupos. O grupo 1 foi composto por 292 (52.7\%) crianças que não fazem treino sistemático e formal de futebol, somente frequentam as aulas de Educação Física no colégio. Estes foram divididos em quatro subgrupos por idade, ou seja, um grupo com indivíduos dos 10 aos 11 anos, um grupo com participantes com idades entre 12 e 13 anos, um grupo com 14 e 15 anos e, por fim, um grupo de 16 e 17 anos de idade. Tais indivíduos são alunos de escolas públicas e particulares do estado do Paraná. O grupo 2 foi composto por $262(47.3 \%)$ atletas praticantes de futebol de campo, também divididos em quatro subgrupos em função da idade, ou seja, grupos de indivíduos com 10-11 anos, 12-13, 14-15, e 16-17 anos. Todos os participantes do grupo 2 são, como referido, atletas de futebol de campo, frequentando centros de formação em futebol há pelo menos 6 meses, e com rotina de treino de, no mínimo, 3 vezes semanais. Todos os atletas são alunos de escolas públicas e particulares do estado do Paraná.

\section{Instrumentos}

\section{Teste de Atenção por Cancelamento}

O Teste de Atenção por Cancelamento (Montiel \& Seabra, 2007) consiste em três matrizes impressas com diferentes tipos de estímulos, sendo a tarefa do sujeito 
assinalar todos os estímulos iguais ao estímulo alvo previamente apresentado. Na primeira parte do teste avalia-se a atenção seletiva, ou seja, a capacidade do individuo para selecionar estímulos-alvo entre distratores. Para tanto, é usada a prova de cancelamento de figuras, cuja matriz tem impressos seis estímulos diferentes (círculo, quadrado, triângulo, cruz, estrela e traço). O estímulo-alvo deve ser assinalado sempre que recorrer e encontrar-se impresso na parte superior da folha, de forma a ficar sempre visível para o sujeito durante a realização da tarefa. O tempo máximo para execução da tarefa é de 1 minuto.

Na segunda parte do teste, o objetivo é avaliar a atenção seletiva mediante uma prova com grau maior de dificuldade. A tarefa consiste em o estímulo alvo ser composto por duplas figuras impressas, na parte superior da folha. O tempo máximo para execução da tarefa é de 1 minuto. Na terceira parte do teste, o objetivo é avaliar a atenção alternada, ou seja, a capacidade do individuo de mudar o foco de atenção de tempos a tempos. Para isso, também é usada uma prova de cancelamento de figuras, na qual o estímulo-alvo muda a cada linha, sendo que a figura inicial de cada linha deve ser considerada o alvo. $\mathrm{O}$ número de vezes que o estímulo-alvo aparece entre as alternativas muda a cada linha, variando de 2 a 6 vezes. O tempo máximo para execução da tarefa é de um minuto. São computados o tempo para a realização da tarefa e três tipos de escores. O primeiro escore corresponde ao número total de acertos, o segundo escore corresponde ao número de erros e o terceiro ao número de omissões.

\section{Avaliação da habilidade motora específica do futebol}

O presente estudo, buscando usar uma bateria que possa ser padronizada para o uso com atletas de futebol, utilizou para a avaliação de habilidades específicas do futebol um protocolo que tem como base a junção de tarefas propostas tanto por Gomes e Erichsen (2004), quanto por Torrelles e Alcaraz (2003). Os primeiros autores são doutores em Educação Física e profissionais do Clube Atlético Paranaense, equipa da primeira divisão do futebol paranaense, que testaram medidas em aproximadamente 5000 mil atletas da categoria de base do clube, desde 2002. Por sua vez, Torrelles e Alcaraz propuseram a Bateria de Testes Aplicados ao Futebol (TAF) para avaliar aspectos físicos, técnicos e táticos do atleta de futebol. Este estudo focalizar-se-à nos testes físicos aplicados ao futebol. Por isso, foi desenvolvido um protocolo para atender a este aspeto da pesquisa reunindo os testes propostos pelos pesquisadores citados.

O protocolo consiste, então, em dez exercícios, para mensuração dos seguintes fatores: Resistência Aeróbia, Flexibilidade, Potência dos Membros Inferiores, Velocidade de Deslocamento, Potência de Membros Superiores, Agilidade, Toques, 
Paredão, Precisão de Passe, Precisão de Chute a Gol em Movimento. A avaliação foi realizada no campo de futebol, utilizando-se bolas de futebol oficiais. Todos os exercícios possuem tabelas de classificação, propostas por Gomes e Erichsen (2004) e Torrelles e Alcaraz (2003).

\section{Procedimento}

Os adolescentes cujos responsáveis consentiram a sua participação na pesquisa foram, então, contatados e, com o consentimento dos próprios adolescentes, foram conduzidos para as sessões de avaliação. Todas as sessões ocorreram na própria instituição durante o período regular das atividades. A dispensa do aluno foi feita pelo professor ou técnico, de forma a não prejudicar as suas atividades desportivas e seu desempenho escolar. Na primeira sessão foi aplicado o Teste de Atenção por Cancelamento (Montiel \& Seabra, 2007), de forma coletiva. Os atletas que foram caracterizados como praticantes de atividade física profissional foram excluídos da pesquisa. O tempo total foi de 15 minutos, tendo o Teste de Atenção por Cancelamento a duração máxima de 3 minutos. Posteriormente, de forma individual, numa segunda sessão, foram aplicados os exercícios para avaliação da habilidade específica do futebol, com tempo de aplicação aproximado entre 30 a 45 minutos.

\section{RESULTADOS}

Inicialmente, para verificar a relação entre a idade e a prática do desporto, foram conduzidas Análises de Variância tendo como variáveis independentes a idade (grupos de 10-11, 12-13, 14-15, e 16-17 anos) e a prática de desporto (atleta e não atleta), e como variáveis dependentes os desempenhos nos instrumentos de avaliação utilizados. Em seguida, para verificar a relação entre os desempenhos nos instrumentos, foram conduzidas análises de correlação de Pearson que se encontram descritas a seguir. Para verificar os desempenhos no Teste de Atenção por Cancelamento (TAC), foram conduzidas análises estatísticas descritivas. A Tabela 1 apresenta valores mínimos, máximos, média e desvio padrão das variáveis do TAC. Nesta análise foram consideradas as medidas número de acertos e número de erros (incluindo itens marcados incorretamente e omissões) nas três partes deste teste. Considerando toda a amostra (atletas e não atletas em todas as faixas etárias), os valores de média de acerto foram 85.62 e intervalo de confiança para 95\% variou entre 83.62 e 87.41 . Para a variável erros, os valores de média foram 
28.09 e o intervalo de confiança para $95 \%$ foi de 25.53 a 30.64 . Considerando a normalidade das medidas obtidas, empregaram-se provas paramétricas para as análises de dados subsequentes.

Na Tabela 1 é possível verificar que em todos os grupos de idade os atletas tiveram desempenho superior aos não atletas, tendo mais acertos e menos erros no TAC. Também se pode verificar que em ambos os grupos há uma disposição para um aumento no número de acertos e queda no número de erros com o aumento da idade. A comparação das medidas por idade foi feita pela ANOVA, adequada para a comparação de mais de três grupos. De facto, a maior parte dos estudos citados na literatura que compara a atenção por idade tende a empregar esta prova, além da prova de correlação de Pearson (Cardoso et al., 2015; García et al., 2011; Andrade et al., 2004; Piek et al., 2004).

Tabela 1

Desempenho dos atletas e não atletas no Teste de Atenção por Cancelamento (TAC), com média de desempenho, desvio padrão e intervalo de confiança para cada grupo e idade.

\begin{tabular}{|c|c|c|c|c|c|c|}
\hline \multirow[b]{2}{*}{ Variável Dependente } & \multirow[b]{2}{*}{ Idade } & \multirow[b]{2}{*}{ Grupo } & \multirow[b]{2}{*}{ Média } & \multirow[b]{2}{*}{ DP } & \multicolumn{2}{|c|}{$\begin{array}{c}\text { Intervalo de Confiança } \\
95 \%\end{array}$} \\
\hline & & & & & $\begin{array}{l}\text { Limite } \\
\text { Inferior }\end{array}$ & $\begin{array}{l}\text { Limite } \\
\text { Superior }\end{array}$ \\
\hline \multirow[t]{8}{*}{ acertos no TAC } & $10-11$ & Atleta & 79.60 & 3.03 & 73.64 & 85.57 \\
\hline & & Não Atleta & 66.65 & 2.86 & 61.02 & 72.28 \\
\hline & $12-13$ & Atleta & 88.97 & 2.90 & 83.26 & 94.68 \\
\hline & & Não Atleta & 85.20 & 2.07 & 81.13 & 89.26 \\
\hline & $14-15$ & Atleta & 91.09 & 2.65 & 85.87 & 96.32 \\
\hline & & Não Atleta & 86.91 & 2.60 & 81.80 & 92.02 \\
\hline & $16-17$ & Atleta & 99.82 & 3.03 & 93.86 & 105.78 \\
\hline & & Não Atleta & 85.92 & 2.49 & 81.02 & 90.81 \\
\hline \multirow[t]{8}{*}{ erros no TAC } & $10-11$ & Atleta & 31.21 & 4.09 & 23.16 & 39.26 \\
\hline & & Não Atleta & 49.81 & 3.86 & 42.21 & 57.41 \\
\hline & $12-13$ & Atleta & 20.92 & 3.92 & 13.21 & 28.62 \\
\hline & & Não Atleta & 29.08 & 2.79 & 23.60 & 34.57 \\
\hline & $14-15$ & Atleta & 22.53 & 3.58 & 15.48 & 29.58 \\
\hline & & Não Atleta & 24.38 & 3.50 & 17.49 & 31.27 \\
\hline & $16-17$ & Atleta & 16.97 & 4.09 & 8.92 & 25.02 \\
\hline & & Não Atleta & 29.82 & 3.36 & 23.21 & 36.42 \\
\hline
\end{tabular}

Quando comparadas as variáveis idade, grupo (atleta e não atleta) e a interação destas em relação aos acertos no TAC, observaram-se diferenças estatisticamente significativas para a variável idade, $\operatorname{com} F(3,339)=17.79, p<.001$. Análises post-hoc de Tukey revelaram que o desempenho da idade na faixa 10 e 11 anos foi signifi- 
cativamente inferior aos das demais idades, com $p<.05$. Ao comparar os atletas com os não atletas, verificou-se desempenho superior para os atletas, $\operatorname{com} F(1,339)$ $=20.45, p<.001$. Não houve, porém, interação entre esses dois fatores $(F(3,339)$ $=2.04, p=.108)$. A Figura 1 representa tais resultados. A prova de Tukey é útil ao usar comparações em pares, e é a melhor quando os tamanhos de amostras não são iguais, e há menos grupos para serem comparados, além de ser o melhor método quando se deseja conhecer intervalos de confiança para as medidas.

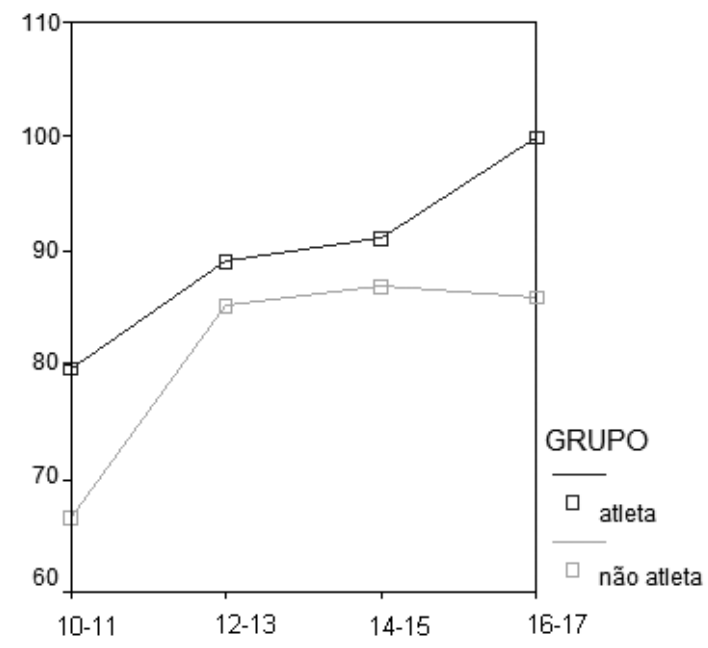

IDADE

Figura 1. Média de acertos no TAC para os grupos de atletas e não atletas, por idade.

Na Figura 1 observa-se uma tendência de crescimento no desempenho em termos de acertos no TAC para ambos os grupos, sendo este mais pronunciado no grupo de 10 e 11 anos em relação aos demais, como a análise de Tukey revelou. Também em conformidade com as análises apresentadas, verifica-se que em todos os grupos de idade os atletas desempenharam melhor que os não atletas.

Quando comparadas as variáveis idade, grupo (atleta e não atleta) e a interação destas em relação aos erros no TAC, incluindo itens errados e omissões, observou-se novamente um efeito de idade, $\operatorname{com} F(3,339)=9.16, p<.001$. Análises post-hoc de Tukey revelaram que o número de erros na idade de 10 e 11 anos foi significativamente superior aos nas demais idades, $\operatorname{com} p<.05$. Ao comparar os atletas com os não atletas verificou-se desempenho superior para os atletas, que cometeram menos erros, $\operatorname{com} F(1,339)=15.92, p<.001$. Porém não houve interação estatisticamente significativa entre as variáveis, $\operatorname{com} F(3,339)=1.80, p=.146)$. Tais resultados encontram-se representados na Figura 2. 


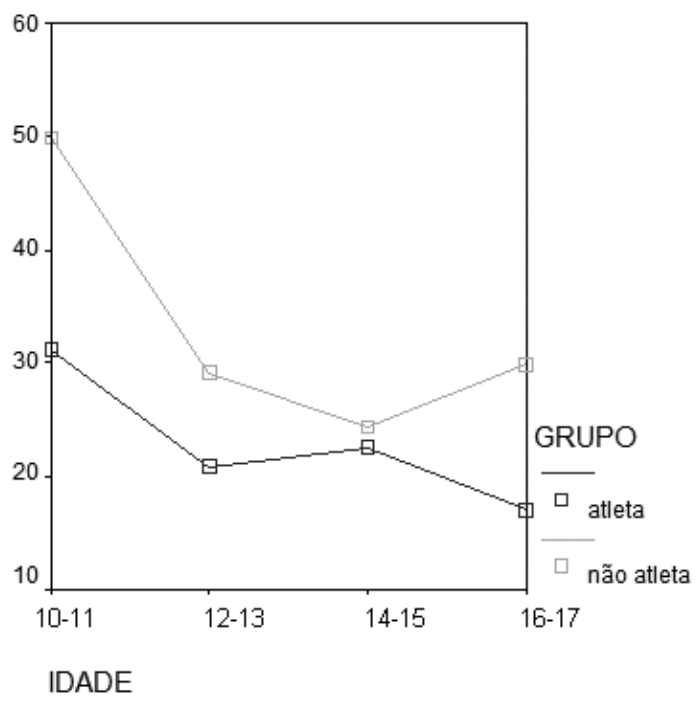

Figura 2. Média de erros no TAC para os grupos de atletas e não atletas, por idade.

Na Figura 2 observa-se que houve uma tendência de queda no número de erros cometidos com o avanço da idade, dado que está de acordo com as análises descritas anteriormente. Porém, a análise de Tukey apenas revelou diferença significativa entre o grupo de 10 e 11 anos e os demais nessa variável. Também nessa figura se pode observar que o grupo de atletas apresentou melhor desempenho, com menor número de erros em todos os grupos de idade.

Para buscar evidência de validade do teste de atenção em relação com o Protocolo de Avaliação de Habilidades Motoras, foram conduzidas análises de correlação de Pearson entre os dois instrumentos, sumariadas na Tabela 2. Como pode ser observado, registam-se correlações significativas, destacadas em negrito, entre o total de acertos no TAC e as seguintes variáveis motoras: toque $(r=-.24, p<.05)$, agilidade $(r=-.30, p<.001)$ e velocidade de deslocamento $(r=-.39, p<.001)$; e entre a percentagem de erros e omissões no TAC e as seguintes variáveis motoras: agilidade $(r=.22, p<.01)$ e velocidade de deslocamento $(r=.29, p<.001)$. 
Tabela 2

Correlações de Pearson, com indicação dos valores de re p, entre as medidas no Teste de Atenção por Cancelamento (TAC) e as variáveis motoras avaliadas no Protocolo de Avaliação de Habilidades Motoras.

\begin{tabular}{|c|c|c|c|}
\hline & & TAC - acertos & TAC - erros e omissões \\
\hline \multirow[t]{2}{*}{ toque } & $r$ & .24 & -.16 \\
\hline & $P$ & .00 & .06 \\
\hline \multirow[t]{2}{*}{ paredão } & $r$ & -.13 & .22 \\
\hline & $P$ & .31 & .08 \\
\hline \multirow[t]{2}{*}{ precisão de passe } & $r$ & .05 & -.01 \\
\hline & $P$ & .56 & .88 \\
\hline \multirow[t]{2}{*}{ precisão de chute a gol (aro direito) } & $r$ & .05 & -.03 \\
\hline & $P$ & .63 & .76 \\
\hline \multirow[t]{2}{*}{ precisão de chute a gol (aro esquerdo) } & $r$ & .10 & -.02 \\
\hline & $P$ & .36 & .87 \\
\hline \multirow[t]{2}{*}{ agilidade } & $r$ & -.30 & .22 \\
\hline & $P$ & .00 & .01 \\
\hline \multirow[t]{2}{*}{ velocidade de deslocamento } & $r$ & -.39 & .29 \\
\hline & $P$ & .00 & .00 \\
\hline \multirow[t]{2}{*}{ flexibilidade } & $r$ & .11 & -.04 \\
\hline & $P$ & .20 & .63 \\
\hline \multirow[t]{2}{*}{ potência membros inferiores } & $r$ & -.10 & .12 \\
\hline & $P$ & .40 & .32 \\
\hline \multirow[t]{2}{*}{ potência membros superiores } & $r$ & .12 & -.06 \\
\hline & $P$ & .18 & .50 \\
\hline \multirow[t]{2}{*}{ resistência aeróbia } & $r$ & -.07 & .06 \\
\hline & $P$ & .54 & .58 \\
\hline
\end{tabular}

\section{DISCUSSÃO}

Considerando as hipóteses inicialmente aventadas, verificou-se uma diferença significativa da atenção entre o grupo de atletas e não atletas, sendo esta maior para o grupo de atletas, confirmando a hipótese formulada e reiterando aspetos já indicados na literatura sobre o tema (Bonilha et al., 2015; Cardoso et al., 2015; Fechine et al., 2015; Fernandes \& Fernandes, 2015; García et al., 2011; Medina-Papst et al., 2016). A segunda hipótese, sobre o efeito de interação entre a idade e o grupo de atletas e não atletas, não foi confirmada, possivelmente pela margem restrita de idades na amostra em questão, não sendo uma amostra com representatividade 
suficiente neste quesito. Sintetizando estes resultados, verificou-se que o desempenho no TAC teve um aumento significativo com a progressão da idade e que o grupo de atletas teve desempenho superior ao de não atletas. Assim, considerando o escore total no teste, verificou-se, sobretudo na pontuação acertos, que o instrumento discriminou entre a idade de 10 e 11 anos e os demais grupos etários. Estes resultados apontam que ocorre melhoria da atenção com o aumento da idade, mas as maiores diferenças são observadas na idade dos 10 e 11 anos em relação aos outros grupos etários.

Alguns autores sugerem que a chegada da puberdade pode influenciar o desempenho das crianças em testes de atenção (Capovilla \& Dias, 2008; Suchomel, 2003). Porém, o presente estudo aponta para um equilíbrio de resultados nas faixas etárias dos 12-13, 14-15 e 16-17 anos, talvez devido ao grau de dificuldade do teste, que pode ser fácil para a presente amostra. Além disso, observou-se que atletas tiveram desempenho superior ao de não atletas, sugerindo maior nível de atenção seletiva nos atletas. Alguns estudos corroboram esse resultado, como os de Ladewig (2000) e Radlo et al. (2002). Cabe ressaltar que mesmo com os achados deste estudo, novas investigações devem ser feitas com amostras mais representativas de idade analisando estas interações. Ao mesmo tempo, trata-se de uma faixa etária em que os processos atencionais e cognitivos tendem a apresentar um plateau e a estabilizarem (Cervelló, 1999; Andreoni et al., 2015). Também, o teste de atenção estudado (TAC) foi desenvolvido e padronizado com crianças mais novas, devendo-se considerar qual a sua adequação e nível de dificuldade, principalmente para os adolescentes. O facto de não terem sido identificados efeitos de interação no grupo de atletas também sugere que o desenvolvimento desta habilidade possa ocorrer de maneira semelhante entre os dois grupos e que, apesar de haver diferenças de atenção entre atletas e não atletas, estas não alteram o rumo do desenvolvimento desta função, embora eleve as médias nestas avaliações. Estudos longitudinais são necessários para sustentar melhor esta posição que extrapola os limites deste estudo transversal.

A terceira hipótese foi confirmada, já que as habilidades físicas que, de facto, demandam atenção se associaram significativamente com os resultados do TAC. Existem estudos sugerindo que a atenção está relacionada com o desempenho de atletas. Por exemplo, Adriano (2003) buscou identificar eventuais variações nos estados atencionais de atletas de futsal em testes aplicados pré e pós-treino. Os resultados revelaram que a pontuação média no teste de atenção aumentou no pós em comparação com o pré-teste, sugerindo um aumento da atenção com o treino. Especificamente em relação ao TAC, os resultados de Barbosa, Capovilla e Calgaro (no prelo) com o objetivo de verificar a existência de validade em relação a outras variáveis de testes (Teste de Atenção Concentrada - AC; Cambraia, 2009, e IDATE - Inventário de Ansiedade 
Traço-Estado; Biaggio \& Natalício, 2009) para avaliar atenção e ansiedade no contexto do desporto, apontaram correlações entre os desempenhos nos dois testes. Houve duas correlações significativas: uma negativa entre percentagem de faltas técnicas e total de acertos no TAC, e outra positiva entre percentagem de erros nos lançamentos e total de erros no TAC. Ou seja, este estudo prévio já havia demonstrado, para outro contexto desportivo, uma relação entre desempenho no TAC e desempenho em situação de jogo, no caso, em atletas femininas jogadoras de andebol.

Em continuidade, bons desempenhos no TAC mostraram-se relacionados com melhor desempenho durante o jogo, em termos de menor número de faltas técnicas e de percentagem de erros de lançamento. Tais resultados são bastante animadores e sugere-se que pesquisas futuras deem continuidade à investigação da validade do TAC na predição do desempenho em jogo.

Os resultados deste estudo contribuem para o estudo de validade do TAC no contexto do desporto. Os resultados encontrados mostram que este teste discriminou adequadamente entre atletas e não atletas. Em relação às correlações, cabe ressaltar que houve correlações significativas entre total de acertos no TAC e as seguintes variáveis motoras: toque, agilidade e velocidade de deslocamento; e entre erros e omissões no TAC e as seguintes variáveis motoras: agilidade e velocidade de deslocamento. Ou seja, bons desempenhos no TAC mostraram-se relacionados com melhor execução nos testes de habilidade motora.

É interessante destacar que as três grandes facetas da avaliação da atenção (manutenção, velocidade e precisão da atenção), apesar de não terem sido adequadamente separadas no instrumento em questão enquanto medidas e possibilidades interpretativas, são explicações possíveis para os dados obtidos, já que os resultados no TAC se relacionam com aspetos motores que envolvem ora velocidade e agilidade, ora maior precisão com o toque. Tal facto abre espaço para novos estudos no âmbito desportivo com este instrumento, visando separar medidas e/ou possibilidades de interpretação destas facetas nos resultados deste teste (Gazzaniga \& Heatherton, 2005).

De facto, esta relação era esperada e é sugerida na literatura. O bom desempenho do atleta é caracterizado por bom desempenho desportivo e está relacionado com um nível elevado de atenção ao jogo e de habilidade motora. Por isso, conforme apresentado na introdução, pesquisas têm evidenciado que atenção e habilidade motora são variáveis correlacionadas (Ladewig, 2000; Magill, 2001; Schmidt \& Wrisberg, 2001; Sternberg, 2000). Por exemplo, segundo Ladewig (2000), a aprendizagem de qualquer habilidade motora requer atenção a informações contidas no meio ambiente e/ou fornecidas pelo professor ou técnico. Deste modo, tal habilidade cognitiva é fundamental à aquisição, estando relacionada com o desempenho motor (Andrade et al., 2004; Piek et al., 2004). 


\section{CONSIDERAÇÕES FINAIS}

De entre as principais limitações deste trabalho pode destacar-se o corte da pesquisa que é transversal, logo as inferências feitas a partir da idade devem ser melhor estudadas com pesquisas longitudinais. Também o tipo de modalidade desportiva é um aspeto que pode apresentar diferenças no grupo de atletas. Assim, a variabilidade intragrupo deve ser melhor explorada em estudos futuros, sobretudo com o TAC. No entanto, os resultados aqui encontrados são bastante semelhantes aos de Piek et al. (2004), segundo os quais a desatenção parece influenciar a variabilidade da execução motora. Os dados deste estudo encontram sustentação em anteriores pesquisas que atestam que o desenvolvimento da cognição está intimamente ligado ao desenvolvimento motor, e ambos são necessários para a criança atingir altos níveis de desempenho desportivo (Campos \& Gallagher, 1991; Campos, Gallagher, \& Ladewig, 1996).

Também o formato de aplicação coletiva do TAC pode ter tido um efeito sobre o tipo dos dados obtidos, já que a aplicação individual pode apresentar menos distratores, quando bem controlada. Todavia, este facto compreende um fator de erro sistemático que pode afetar a média da distribuição como um todo, sendo de difícil controlo neste caso e convidando a outros tipos de formatos de coleta para comparar os resultados. Vale ressaltar que na maior parte das pesquisas com este instrumento procedeu-se a uma aplicação coletiva, mas com crianças e não com adolescentes, restando novos espaços para outros estudos.

Finalmente, os dados obtidos fornecem boas evidências de validade para o TAC, sendo apropriado para avaliação da atenção em atletas, já que as relações indicadas na literatura, sobre atenção em atletas, foram observadas no caso deste estudo com o TAC. Outras possibilidades de interpretação dos escores deste teste devem ser exploradas em atletas considerando as diferenças entre as três grandes facetas da atenção (manutenção, velocidade e precisão do desempenho) restando espaço para futuras investigações.

\section{REFERÊNCIAS}

Abes, L. O. (2004). Diferença entre o foco de atenção interno e externo, frequência cardíaca e desempenho no primeiro saque de tênis em jogadores iniciantes, intermediários e avançados (Tese de Mestrado não publicada). Universidade Federal de Santa Catarina, Santa Catarina, Brasil.

Adriano, N. (2003). Avaliando o nível de concentração e atenção de atletas de futsal através de testes pré e pós-treinamentos. Revista Digital-Buenos Aires, 9(63). Disponível em http:www.efdeportes. com/efd63/teste.htm/ [Acesso em: 02-03-1997]. 
Andrade A., Luft, B. C., \& Rolim, B. S. K. M (2004). O desenvolvimento motor, a maturação das áreas corticais e a atenção na aprendizagem motora. Revista Digital-Buenos Aires, 10(78), 1-14. Disponível em http://www.efdeportes.com/ [Acesso em: 11-2004].

Andreoni, J., Kuhn, M. A., \& Sprenger, C. (2015). Measuring time preferences: A comparison of experimental methods. Journal of Economic Behavior \& Organization, 116, 451-464. Doi: 10.1016/j.jebo.2015.05.018

Barbosa, C., Capovilla, A. G. S., \& Calgaro, D. (no prelo). Validade de testes de atenção e ansiedade na predição do desempenho no handebol. Revista Thêma et Scientia, 1(2), 130-141..

Becker Jr., B. (2000). Manual de psicologia do esporte e exercício. Porto Alegre: Novaprova.

Bertolucci, P. H. F. (2000). Demência em jovens: exame inicial e causas mais comuns. Psicologia: Teoria e Prática, 2(2), 31-42.

Biaggio, A. M. B., \& Natalício, L. (1979). Manual para o Inventário de Ansiedade Traço Estado (IDATE). Rio de Janeiro: CEPA.

Bonilla, P. U., Romero, L. B., Sánchez-Ureña, B., \& Salas-Cabrera, J. (2015). Caracterización psicológica y autovaloración del rendimiento en jugadores de fútbol y baloncesto en la primera división costarricense. Cuadernos de Psicología del Deporte, 15(2), 13-20.

Cambraia, S. V. (2003). Teste de Atenção Concentrada - AC. São Paulo: Vetor Editora Psicopedagogica. Campos, W., \& Gallagher, J. D. (1991). Knowledge base and sport skill performance. Paper presented at the Meeting of the Americam Aliance for Heath, Phisical Education, Recreation and Dance, San Francisco, CA.

Campos, W., Gallagher, J. D., \& Ladewig, I. (1996). Os efeitos da idade e nível de experiência na performance cognitiva e motora em crianças praticantes de futebol. Revista Synopsis, 7(7), 13-22.

Capovilla, A. G. S., Capovilla, F. C., \& Macedo, E. C. (2005). Teste de geração semântica computadorizado. Programa de computador. Itatiba: Universidade São Francisco.

Capovilla, A. G. S., \& Dias, N. M. (2008). Desenvolvimento de habilidades atencionais em estudantes da $1^{\text {a }}$ à $4^{\mathrm{a}}$ série do ensino fundamental e relação com rendimento escolar. Psicopedagogia, 25(78), 1-14.

Capovilla, A. G. S., Montiel, J. M., Macedo, E. C., \& Charin, S. (2005). Teste de Stroop Computadorizado. Programa de computador. Itatiba: Universidade São Francisco.

Cardoso, F., Machado, G., \& Teoldo, I. (2015). Relação entre impulsividade e comportamento tático de jogadores de futebol sub-11. Psicologia: Teoria e Prática, 17(1), 108-119. Doi: 10.15348/19806906/psicologia.v17n1p108-119

Cervelló, E. M. G. (1999). Introducción al entrenamiento psicológico. In J. P. F. García (Org.). Enseñanza y entrenamiento del tenis. (pp.145-182). Cáceres: Servicio de puclicaciones da Universidad de Extremadura.

Covre, P., Macedo, E. C., Capovilla, F. C., \& Schwartzman, J. S. (2005). Movimentos oculares e padrões de busca visual em tarefas de rotação mental. Psico-USF, 10(1), 41-50.

Covre, P., Piza, C. M. J. T., Lukasova, K., \& Macedo, E. C. (2002). Diferenças de gênero na capacidade de rotação mental de objetos. Boletim de Iniciação Científica em Psicologia da Universidade Presbiteriana Mackenzie, 3(1), 19-26.

Cozza, H. F. P. (2005). Avaliação das funções executivas em crianças e correlação com atenção hiperatividade (Tese de Mestrado não publicada). Universidade São Francisco, Itatiba, Brasil.

Cozza, H. F. P. (2008). Avaliação neuropsicológica das funções executivas em atletas e correlação com desempenho em situação de jogo (Tese de Doutoramento não publicada). Universidade São Francisco, Itatiba, Brasil.

Dalgalarrondo, P. (2000). Psicopatologia e semiologia dos transtornos mentais. Porto Alegre: Artes Médicas. 
Fechine, B., Costa, A., Vasconcelos, O., Botelho, M., \& Carvalho, J. (2015). Cognição e atividade física: a relação existente entre atenção e a velocidade perceptiva em idosas praticantes e não praticantes de atividade física. Inter Science Place, 1(27), 120-138.

Fernandes, M. G., \& Fernandes, H. M. (2015). Tradução, adaptação transcultural e propriedades psicométricas da versão brasileira do Test of Performance Strategies. Psicologia: Reflexão e Crítica, 28(1), 136-146. doi: 10.1590/1678-7153.201528115

García, S., Rodríguez, A., \& Garzón, A. (2011). Conceptualización de inteligencia táctica en fútbol: Consideraciones para el desarrollo de un instrumento de evaluación en campo desde las funciones ejecutivas. Cuadernos de Psicología del Deporte, 11(1), 69-78.

Gazzaniga, M. S., \& Heatherton, T. F. (2005). Ciência psicológica: mente, cérebro e comportamento. Porto Alegre: Artmed.

Gomes, A C., \& Erichsen, O. A. (2004). Preparação de futebolistas na infância e adolescência. In I. Guerra \& T. L. Barros (eds.), Ciência do futebol (pp. 241-275). Barueri: Manole.

Gould, D., \& Damarjian, N. (2000). Treinamento mental no esporte. In B. Elliott \& J. Mester (eds.), Treinamento no esporte: Aplicando ciências no esporte (pp. 99-152). São Paulo: Phorte Editora.

Guallar, A., \& Pons, D. (1994). Concentración y atención en el deporte. In I. Balaguer (ed.), Entrenamiento psicológico en el deporte (pp. 207-245). Valencia: Albatros Educación.

Harle, S. K., \& Vickers, J. N. (2001). Training quiet eye improves accuracy in the basketball three throw. The Sport Psychologist. 15(3), 289-305. Doi: 10.1123/tsp.15.3.289

Ladewig, I. (2000). A importância da atenção na aprendizagem de habilidades motoras. Revista Paulista de Educação Física, Supl. 3, 62-71.

Liao, C., \& Masters, R. S. W. (2002). Self-focused attention and performance failure under psychological stress. Journal of Sport \& Exercise Psychology, 24, 289-305.

Magill, R. A. (2001). Aprendizagem motora: Conceitos e aplicações (5a ed.). São Paulo: Edgard Blucher. Martens, R. (1987). Coaches guide to sport psychology. Champaign, IL: Human Kinetics.

Medina-Papst, J., Bordini, F. L., \& Marques, I. (2016). Instruções de foco de atenção para a automatização da ação na aprendizagem de uma habilidade manipulativa. Motricidade, 11(4), 36-46.

Montiel, J. M. (2005). Evidências de Validade de instrumentos para Avaliação Neuropsicológica do Transtorno de Pânico (dissertação de Mestrado não publica). Universidade São Francisco - USF, Brasil.

Montiel, J. M., \& Capovilla, A. G. S. (2007). Teste de Atenção por Cancelamento. In A. G. S. Capovilla \& F. C. Capovilla (Eds.), Teoria e pesquisa em avaliação neuropsicológica (pp. 119-124). São Paulo: Memnon.

Moraes, L. C. (1990). Ansiedade e desempenho no ssporte. Revista Brasileira de Ciência e Movimento, 4(2), 51-56.

Nideffer, R. M. (1976). Test of Attentional and Interpersonal Style. Journal of Personality and Social Psychology, 34, 394-404.

Piek, J. P., Dyck, M. J., Nieman, A., Anderson, M., Hay, D., Smith, L. M., Mccoy M., \& Hallmayer, J. (2004). The relationship between motor coordination, executive functioning and attention in school aged children. Archives of Clinical Neuropsychology, 19(8), 1063-1076.

Primi, R. (2002). Teste de memória de trabalho. Programa de computador. Itatiba: Universidade São Francisco - LabAPE.

Radlo, S. J., Steinberg, G. M., Singer, R. N., Barba, D. A., \& Melnikov, A. (2002). The influence of an attentional focus strategy on alpha brain wave activity, heart rate, and dartthrowing performance. International Journal of Sport Psychology, 33(2), 205-217. 
Robazza, C., Bortoli, L., \& Nougier, V. (1998). Physiological arousal and performance in elite archers: A field study. European psychologist, 3(4), 263-270.

Samulski, D. M. (2002). Psicologia do esporte. Barueri: Manole.

Sarter, M., Givens, B., \& Bruno, J. P. (2001). The cognitive neuroscience of sustained attention: where top-down meets bottom-up. Brain Research Reviews, 35(2), 146-160.

Schmidt R. A., \& Wrisberg C. A (2001). Aprendizagem e performance motora: Uma abordagem da aprendizagem baseada no problema (2a ed.). Porto Alegre: Artmed.

Shea, C. H., \& Wulf, G. (1999). Enhancing motor learning through external-focus instructions and feedback. Human movement Science, 18, 553-571.

Solanellas, F., Front, J., \& Rodríguez, F. A. (1996). Prevalencia del estilo atencional en la población catalana de tensitas. Apunts Educación Física y deportes, 44-45, 154-163.

Sternberg, R. J. (2000). Psicologia cognitiva. Porto Alegre: Artmed.

Suchomel, A. (2003). The biological age of prepubescent and pubescent children with low and high motor efficiency. Anthropologischer Anzeiger, 61(1), 67-77.

Thomas, P. R., Murphy, S. M., \& Hardy, L. (1999). Test of Performance Strategies: Development and preliminary validation of a comprehensive measure of athletes' psychological skills. Journal of Sports Sciences, 17(9), 697-711.

Torrelles, A. S., \& Alcaraz C. F. (2003). Escolas de futebol: Manual para organização e treinamento. Porto Alegre: Artmed.

Vicker, J. N., Rodrigues S. T., \& Brown, L. N. (2002). Gaze pursuit and arm control of adolescents males diagnosed with attention deficit disorder (ASHD) and normal controls: Evidence of dissociation in processing visual information of short and long duration. Journal of Sport Sciences. 20(3), 201-216.

Vickers, J. N. (1996). Control of visual attention during the basketball three throw. The American Journal of Sport Medicine. 24(6), 94-97.

Weinberg, R., \& Gould, D. (2001). Fundamentos da psicologia do esporte e do exercício. Porto Alegre: Artmed.

Wulf, G., McConnel, N., Gärtner, M., \& Schwarz, A. (2002). Enhancing the learning sport skills through external-focus feedback. Journal of Motor Behavior, 34(2), 171-182. 